\title{
Generalized Topological Notions by Operators
}

\author{
Ismail Ibedou* \\ Department of Mathematics, Faculty of Science, Benha University, Benha 13518, Egypt
}

\begin{abstract}
In this paper, it is introduced the notion of $r$-fuzzy $\beta-T_{i}, i=0,1,2$ separation axioms related to a fuzzy operator $\beta$ on the initial set $X$ which is a generalization of previous fuzzy separation axioms. An $r$-fuzzy $\alpha$-connectedness related to a fuzzy operator $\alpha$ on the set $X$ is introduced which is a generalization of many types of $r$-fuzzy connectedness. An $r$-fuzzy $\alpha$-compactness related to a fuzzy operator $\alpha$ on the set $X$ is introduced which is a generalization of many types of fuzzy compactness.
\end{abstract}

Keywords: fuzzy operators; fuzzy separation axioms; fuzzy compactness; fuzzy connectedness.

(2000 Mathematics Subject Classification: 54A40, 54A05, 54C10, 54D10, 54D30)

\section{Introduction and Preliminaries}

It is a way to use fuzzy operators $\alpha, \beta$ on the initial set $X$ and to use fuzzy operators $\theta, \delta$ on the set $Y$ giving generalizations of many notions and results in fuzzy topological spaces. $r$-fuzzy $\beta-T_{i}, i=0,1,2$ separation axioms of the set $X$ is a new type of fuzzy separation axioms related with a fuzzy operator $\beta$ on $X$. It is proved that the image of $r$-fuzzy $\beta$ - $T_{i}, i=0,1,2$ is $r$-fuzzy $\delta$ - $T_{i}, i=0,1,2$, and also the preimage of $r$-fuzzy $\delta$ $T_{i}, i=0,1,2$ is $r$-fuzzy $\beta$ - $T_{i}, i=0,1,2$. $r$-fuzzy $\alpha$-connectedness is introduced related with the fuzzy operator $\alpha$ on $X$ giving a generalization of many of fuzzy connectedness notions. It is proved that the image of $r$-fuzzy $\alpha$-connected is $r$-fuzzy $\theta$-connected, and some particular cases are included. $r$-fuzzy $\alpha$-compactness is introduced using the fuzzy operator $\alpha$ on $X$ giving a generalization of many of fuzzy compactness notions. It is proved that the image of $r$-fuzzy $r$-fuzzy compact is $r$-fuzzy $\theta$-compact, and many special cases are deduced.

Throughout the paper, $X$ refers to an initial universe, $I^{X}$ is the set of all fuzzy sets on $X$ (where $I=[0,1], I_{0}=(0,1], \lambda^{c}(x)=1-\lambda(x) \forall x \in X$ and for all $t \in I$, $\bar{t}(x)=t \forall x \in X)$.

$(X, \tau)$ is a fuzzy topological space $([1])$, if $\tau: I^{X} \rightarrow I$ satisfies the following conditions:

(O1) $\tau(\overline{0})=\tau(\overline{1})=1$,

\footnotetext{
*Department of Mathematics, Faculty of Science, Jazan University, Saudi Arabia e-mail: ismail.ibedou@gmail.com, iibedou@jazanu.edu.sa
} 
(O2) $\tau\left(\lambda_{1} \wedge \lambda_{2}\right) \geq \tau\left(\lambda_{1}\right) \wedge \tau\left(\lambda_{2}\right)$ for all $\lambda_{1}, \lambda_{2} \in I^{X}$,

(O3) $\tau\left(\bigvee_{j \in J} \lambda_{j}\right) \geq \bigwedge_{j \in J} \tau\left(\lambda_{j}\right)$ for all $\left\{\lambda_{j}\right\}_{j \in J} \subseteq I^{X}$.

By the concept of a fuzzy operator on a set $X$ is meant a map $\gamma: I^{X} \times I_{0} \rightarrow I^{X}$. Assume with respect to a fuzzy topology in $\check{S}$ ostak sense defined on $X$, we have

$$
\operatorname{int}_{\tau}(\mu, r) \leq \gamma(\mu, r) \leq \operatorname{cl}_{\tau}(\mu, r) \quad \forall \mu \in I^{X}, \forall r \in I_{0},
$$

where $\operatorname{int}_{\tau}, \mathrm{cl}_{\tau}: I^{X} \times I_{0} \rightarrow I^{X}$ are defined in $\check{S}$ ostak sense for any $\mu \in I^{X}$ and each grade $r \in I_{0}$ as follows:

$$
\begin{aligned}
& \operatorname{int}_{\tau}(\mu, r)=\bigvee\left\{\eta \in I^{X}: \eta \leq \mu, \tau(\eta) \geq r\right\} \text { and } \\
& \operatorname{cl}_{\tau}(\mu, r)=\bigwedge\left\{\eta \in I^{X}: \eta \geq \mu, \tau\left(\eta^{c}\right) \geq r\right\} .
\end{aligned}
$$

Let $\left(X, \tau_{1}\right)$ and $\left(Y, \tau_{2}\right)$ be two fuzzy topological spaces, $\alpha$ and $\beta$ are fuzzy operators on $X, \theta$ and $\delta$ are fuzzy operators on $Y$, respectively. This type of maps $\alpha$ or $\beta$ is called an expansion on $X$ or a fuzzy operator on $\left(X, \tau_{1}\right)$, and the map $\theta$ or $\delta$ is called an expansion on $Y$ or a fuzzy operator on $\left(Y, \tau_{2}\right)$.

Recall that a fuzzy ideal $\mathcal{I}$ on $X([2])$ is a map $\mathcal{I}: I^{X} \rightarrow I$ that satisfies the following conditions:

(1) $\lambda \leq \mu \Rightarrow \mathcal{I}(\lambda) \geq \mathcal{I}(\mu)$

(2) $\mathcal{I}(\lambda \vee \mu) \geq \mathcal{I}(\lambda) \wedge \mathcal{I}(\mu)$

Also, $\mathcal{I}$ is called proper if $\mathcal{I}(\overline{1})=0$ and there exists $\mu \in I^{X}$ such that $\mathcal{I}(\mu)>0$. Define the fuzzy ideal $\mathcal{I}^{\circ}$ by

$$
\mathcal{I}^{\circ}(\mu)= \begin{cases}1 & \text { at } \mu=\overline{0} \\ 0 & \text { otherwise }\end{cases}
$$

Let us define the fuzzy difference between two fuzzy sets as follows:

$$
(\lambda \bar{\wedge} \mu)= \begin{cases}\overline{0} & \text { if } \lambda \leq \mu, \\ \lambda \wedge \mu^{c} & \text { if } \lambda \leq \leq \mu .\end{cases}
$$

Let us fix that:

(1) $\beta$ is a fuzzy operator on $X$ such that $\beta(\mu, r) \leq \mu \forall \mu \in I^{X}, \forall r \in I_{0}$.

(2) $\alpha$ is a fuzzy operator on $X$ such that $\alpha(\mu, r) \geq \mu \forall \mu \in I^{X}, \forall r \in I_{0}$.

As a special case of fuzzy operators, by the identity fuzzy operator $i d_{X}$ on a set $X$ we mean that $i d_{X}: I^{X} \times I_{0} \rightarrow I^{X}$ so that $i d_{X}(\nu, r)=\nu \forall \nu \in I^{X}, \forall r \in I_{0}$. 


\section{Definition 1.1}

(1) A mapping $f:\left(X, \tau_{1}\right) \rightarrow\left(Y, \tau_{2}\right)$ is said to be fuzzy $(\alpha, \beta, \theta, \delta, \mathcal{I})$-continuous if for every $\mu \in I^{Y}$, any fuzzy ideal $\mathcal{I}$ on $X$,

$$
\mathcal{I}\left[\alpha\left(f^{-1}(\delta(\mu, r)), r\right) \bar{\wedge} \beta\left(f^{-1}(\theta(\mu, r)), r\right)\right] \geq \tau_{2}(\mu) ; r \in I_{0} .
$$

We can see that the above definition generalizes the concept of fuzzy continuity ([1]) when we choose $\alpha=$ identity operator, $\beta=$ interior operator, $\delta=$ identity operator, $\theta=$ identity operator and $\mathcal{I}=\mathcal{I}^{\circ}$.

(2) A mapping $f:\left(X, \tau_{1}\right) \rightarrow\left(Y, \tau_{2}\right)$ is said to be fuzzy $\left(\alpha, \beta, \theta, \delta, \mathcal{I}^{*}\right)$-open if for every $\lambda \in I^{X}$, any fuzzy ideal $\mathcal{I}^{*}$ on $Y$,

$$
\mathcal{I}^{*}[\theta(f(\beta(\lambda, r)), r) \bar{\wedge} \delta(f(\alpha(\lambda, r)), r)] \geq \tau(\lambda) ; r \in I_{0}
$$

We can see that the above definition generalizes the concept of fuzzy openness ([1]) when we choose $\alpha=$ identity operator, $\beta=$ interior operator, $\delta=$ interior operator, $\theta=$ identity operator and $\mathcal{I}^{*}=\mathcal{I}^{\circ}$.

\section{2. $r$-fuzzy $\beta-T_{i}$ separation axioms}

Here, we introduce and study fuzzy separation axioms related with a fuzzy operator $\beta$ on the initial set $X$.

\section{Definition 2.1}

(1) A set $X$ is called $r$-fuzzy $\beta$ - $T_{0}$ if for all $x \neq y$ in $X$, there exists $\lambda \in I^{X}, r \in I_{0}$ with $t \leq \beta(\lambda, r)(x) ; t \in I_{0}$ such that $t>\lambda(y)$ or there exists $\mu \in I^{X}, r \in I_{0}$ with $s \leq \beta(\mu, r)(y) ; s \in I_{0}$ such that $s>\mu(x)$.

(2) A set $X$ is called $r$-fuzzy $\beta$ - $T_{1}$ if for all $x \neq y$ in $X$, there exist $\lambda, \mu \in I^{X}, r \in I_{0}$ with $t \leq \beta(\lambda, r)(x), s \leq \beta(\mu, r)(y) ; t, s \in I_{0}$ such that $t>\lambda(y), s>\mu(x)$.

(3) A set $X$ is called $r$-fuzzy $\beta$ - $T_{2}$ if for all $x \neq y$ in $X$, there exist $\lambda, \mu \in I^{X}, r \in I_{0}$ with $t \leq \beta(\lambda, r)(x), s \leq \beta(\mu, r)(y) ; t, s \in I_{0}$ such that $(t \wedge s)>\sup (\lambda \wedge \mu)$.

Proposition 2.1 Every $r$-fuzzy $\beta-T_{i}$ set $X$ is an $r$-fuzzy $\beta-T_{i-1}, \quad i=1,2$.

\section{Proof.}

$r$-fuzzy $\beta-T_{2} \Rightarrow r$-fuzzy $\beta-T_{1}$ : Suppose that $X$ is an $r$-fuzzy $\beta-T_{2}$ but it is not $r$-fuzzy $\beta-T_{1}$. Then, for all $x \neq y$ in $X$ and for all $\lambda \in I^{X}$ with $t \leq \beta(\lambda, r)(x), r \in I_{0}$, 
suppose that $\lambda(y) \geq t ; t \in I_{0}$. Now, for $\mu \in I^{X}$ with $s \leq \beta(\mu, r)(y) \leq \mu(y) ; s \in I_{0}$, we get that

$$
\sup (\lambda \wedge \mu) \geq(\lambda \wedge \mu)(y) \geq(t \wedge s),
$$

which means a contradiction to $X$ is $r$-fuzzy $\beta-T_{2}$. Hence, $X$ is an $r$-fuzzy $\beta-T_{1}$.

$r$-fuzzy $\beta-T_{1} \Rightarrow r$-fuzzy $\beta-T_{0}$ : Direct.

Recall that: a fuzzy operator $\theta$ is finer than a fuzzy operator $\beta$ on a set $X$, denoted by $\beta \sqsubseteq \theta$, if $\beta(\nu, r) \leq \theta(\nu, r) \quad \forall \nu \in I^{X}, \forall r \in I_{0}$.

Proposition 2.2 Let $X$ be an $r$-fuzzy $\beta-T_{i}, i=0,1,2$, and $\theta$ a fuzzy operator on $X$ finer than $\beta$. Then $X$ is also $r$-fuzzy $\theta-T_{i}$ space, $i=0,1,2$.

Proof. For all the axioms $r$-fuzzy $\beta-T_{i}, i=0,1,2$, the proof comes from that $\beta(\nu, r) \leq$ $\theta(\nu, r) \forall \nu \in I^{X}, \forall r \in I_{0}$.

\section{Example 2.1}

(1) Let $X=\{x, y\}, r \in I_{0}$ and

$$
\beta(\nu, r)= \begin{cases}\nu & \text { at } \nu=\overline{0}, \overline{1} \\ x_{1} & \text { at } x_{1} \leq \nu<\overline{1} \\ \overline{0} & \text { otherwise }\end{cases}
$$

Then, we get $\lambda=x_{1} \in I^{X}, t=\frac{1}{4} \in I_{0}$ with $\beta(\lambda, r)(x)=x_{1}(x)=1 \geq t$ and $\lambda(y)=x_{1}(y)=0<t$. Hence, the set $X$ is an $r$-fuzzy $\beta$ - $T_{0}$ set.

(2) Let $X=\{x, y\}, r \in I_{0}$. In case of

$$
\beta(\nu, r)= \begin{cases}\nu & \text { at } \nu=\overline{0}, \overline{1} \\ \overline{0} & \text { otherwise. }\end{cases}
$$

It could not be found $\lambda \in I^{X}$ such that $\lambda(y)<t \leq \beta(\lambda, r)(x) ; t \in I_{0}$, and also you could not find $\mu \in I^{X}$ such that $\mu(x)<s \leq \beta(\mu, r)(y) ; s \in I_{0}$ and thus, the set $X$ is not $r$-fuzzy $\beta$ - $T_{0}$ set.

(3) $X$ as given in (1), (2) is not $r$-fuzzy $\beta-T_{1}$ set and is not $r$-fuzzy $\beta-T_{2}$ set.

(4) Let $X=\{x, y\}, r \in I_{0}$ and

$$
\beta(\nu, r)= \begin{cases}\nu & \text { at } \nu=\overline{0}, \overline{1} \\ x_{1} & \text { at } x_{1} \leq \nu<\overline{1} \\ y_{1} & \text { at } y_{1} \leq \nu<\overline{1} \\ \overline{0} & \text { otherwise }\end{cases}
$$


Then, we get $\lambda=y_{1} \in I^{X}, t=\frac{1}{5} \in I_{0}$ with $\beta(\lambda, r)(y)=y_{1}(y)=1 \geq t$ and $\lambda(x)=y_{1}(x)=0<t$. Similarly, we get $\mu=x_{1} \in I^{X}, s=\frac{1}{3} \in I_{0}$ with $\beta(\mu, r)(x)=x_{1}(x)=1 \geq s$ and $\mu(y)=x_{1}(y)=0<s$. Hence, the set $X$ is an $r$-fuzzy $\beta$ - $T_{1}$ set.

For $\lambda=x_{1} \vee y_{\frac{1}{2}}, \mu=y_{1} \vee x_{\frac{1}{2}} \in I^{X}, t, s>\frac{1}{2} \in I_{0}$, we get that

$$
\beta(\lambda, r)(x)=x_{1}(x)=1 \geq t \text { and } \beta(\mu, r)(y)=y_{1}(y)=1 \geq s
$$

such that

$$
(t \wedge s)>\frac{1}{2}=\sup \left(x_{\frac{1}{2}} \vee y_{\frac{1}{2}}\right)=\sup (\lambda \wedge \mu) .
$$

Hence, the set $X$ is an $r$-fuzzy $\beta-T_{2}$ set.

(5) Let $X=\{x, y\}, r \in I_{0}$ and

$$
\beta(\nu, r)= \begin{cases}\nu & \text { at } \nu=\overline{0}, \overline{1} \\ \overline{0.2} & \text { at } \overline{0.2} \leq \nu<\overline{0.4} \\ \overline{0.4} & \text { at } \overline{0.4} \leq \nu<\overline{1} \\ \overline{0} & \text { otherwise }\end{cases}
$$

Then,

(i) For all $\lambda=\bar{p}, 0.4 \leq p<1$, we get that: $\beta(\lambda, r)(x)=\overline{0.4}(x)=0.4 \geq t$ for $t \leq 0.4 \in I_{0}$ and $\beta(\lambda, r)(y)=\overline{0.4}(y)=0.4 \geq s$ for $s \leq 0.4 \in I_{0}$.

(ii) For all $\lambda=\bar{p}, 0.2 \leq p<0.4$, we get that: $\beta(\lambda, r)(x)=\overline{0.2}(x)=0.2 \geq t$ for $t \leq 0.2 \in I_{0}$ and $\beta(\lambda, r)(y)=\overline{0.2}(y)=0.2 \geq s$ for $s \leq 0.2 \in I_{0}$.

(iii) For all $\lambda=\bar{p}, p<0.2$, and for any $\lambda=x_{p}$ or $\lambda=y_{q}, p, q \in I$, we get that: $\beta(\lambda, r)(x)=\overline{0}(x)=0=\overline{0}(y)=\beta(\lambda, r)(y)$.

So, we care about the first two cases. If $\lambda, \mu \in I^{X}$ are of type (i), then $(t \wedge s) \leq$ $0.4 \leq \sup (\lambda \wedge \mu)$, and if $\lambda, \mu$ are of type (ii), then $(t \wedge s) \leq 0.2 \leq \sup (\lambda \wedge \mu)$, and if $\lambda$ is of type (i) and $\mu$ is of type (ii) or the converse, then $(t \wedge s) \leq$ $0.4 \wedge 0.2=0.2 \leq \sup (\lambda \wedge \mu)$. Hence, for every $\lambda, \mu \in I^{X}$ with $\beta(\lambda, r)(x) \geq t$ and $\beta(\mu, r)(y) \geq s ; t, s \in I_{0}$, we have $(t \wedge s) \leq \sup (\lambda \wedge \mu)$ and thus, $X$ is not an $r$-fuzzy $\beta-T_{2}$ set.

(6) Let $X=\{x, y\}, r \in I_{0}$ and

$$
\beta(\nu, r)= \begin{cases}\nu & \text { at } \nu=\overline{0}, \overline{1} \\ \overline{0.2} & \text { at } \overline{0.2} \leq \nu, \nu<x_{1} \vee y_{0.2}, \nu<x_{0.2} \vee y_{1} \\ x_{1} \vee y_{0.2} & \text { at } x_{1} \vee y_{0.2} \leq \nu<\overline{1} \\ x_{0.2} \vee y_{1} & \text { at } x_{0.2} \vee y_{1} \leq \nu<\overline{1} \\ \overline{0} & \text { otherwise. }\end{cases}
$$


Then, there exist $\lambda=x_{1} \vee y_{0.3}, \mu=x_{0.3} \vee y_{1}$ such that $\beta(\lambda, r)(x)=1 \geq t>$ $0.3=\lambda(y)$ for $t \in I_{0}$ and $\beta(\mu, r)(y)=1 \geq s>0.3=\mu(x)$ for $s \in I_{0}$, and then $X$ is an $r$-fuzzy $\beta$ - $T_{1}$ set.

Now, we study all possible fuzzy sets in $I^{X}$ :

Then

(a) For any $\lambda=x_{1} \vee y_{p}, \mu=x_{1} \vee y_{q}, p, q \geq 0.2$, we get that: $\beta(\lambda, r)(x)=1 \geq$ $t, \beta(\mu, r)(y)=0.2 \geq s ; t, s \in I_{0}$ but $(t \wedge s) \leq 0.2 \leq \sup (\lambda \wedge \mu), p, q \geq 0.2$.

(b) For any $\lambda=x_{p} \vee y_{1}$ or $x_{1} \vee y_{p}, \mu=x_{q} \vee y_{1}$ or $x_{1} \vee y_{q}, p, q<0.2$, we get that: $\beta(\lambda, r)(x)=\overline{0}(x)=0=\overline{0}(y)=\beta(\mu, r)(y)$.

(c) For any $\lambda=x_{p}, \mu=x_{q}$ or $\lambda=y_{p}, \mu=y_{q}$ or $\lambda=x_{p}, \mu=y_{q}, p, q \in I$, we get that: $\beta(\lambda, r)(x)=\overline{0}(x)=0=\overline{0}(y)=\beta(\mu, r)(y)$.

Hence, for every $\lambda, \mu \in I^{X}$ with $\beta(\lambda, r)(x) \geq t$ and $\beta(\mu, r)(y) \geq s ; t, s \in I_{0}$, we have $(t \wedge s) \leq \sup (\lambda \wedge \mu)$, and thus $X$ is not an $r$-fuzzy $\beta-T_{2}$ set.

Proposition 2.3 Let $f: X \rightarrow Y$ be an injective mapping. Assume that $\delta$ is a fuzzy operator on $Y$ such that

$$
f^{-1}(\delta(\lambda, r)) \leq \beta\left(f^{-1}(\lambda), r\right) \quad \forall \lambda \in I^{Y}, \forall r \in I_{0} .
$$

Then, $Y$ is an $r$-fuzzy $\delta-T_{i}$ implies that $X$ is an $r$-fuzzy $\beta-T_{i}, \quad i=0,1,2$.

\section{Proof.}

Since $x \neq y$ in $X$ implies that $f(x) \neq f(y)$ in $Y$ and $Y$ is an $r$-fuzzy $\delta$ - $T_{1}$, then there exists $\lambda \in I^{Y}$ with $t \leq \delta(\lambda, r)(f(x)) ; t \in I_{0}$ so that $t>\lambda(f(y))$, that is,

$$
t \leq\left[f^{-1}(\delta(\lambda, r))\right](x) \leq\left[\beta\left(f^{-1}(\lambda), r\right)\right](x) \text { and } t>\left(f^{-1}(\lambda)\right)(y)
$$

which means that there exists $\mu=f^{-1}(\lambda) \in I^{X}$ with $t \leq \beta(\mu, r)(x) ; t \in I_{0}$ so that $t>\mu(y)$. Hence, $X$ is an $r$-fuzzy $\beta-T_{1}$, and consequently $X$ is an $r$-fuzzy $\beta-T_{0}$.

Now, for $x \neq y$ in $X$ implies that $f(x) \neq f(y)$ in $Y$ and $Y$ is an $r$-fuzzy $\delta-T_{2}$, then there exist $\lambda, \mu \in I^{Y}$ with $t \leq \delta(\lambda, r)(f(x)), s \leq \delta(\mu, r)(f(y)) ; s, t \in I_{0}$ so that $(t \wedge s)>\sup (\lambda \wedge \mu)$.

Since $\sup (\lambda \wedge \mu) \geq \sup \left(f^{-1}(\lambda) \wedge f^{-1}(\mu)\right)$, then $(t \wedge s)>\sup \left(f^{-1}(\lambda) \wedge f^{-1}(\mu)\right)$. Also,

$t \leq\left[f^{-1}(\delta(\lambda, r))\right](x) \leq\left[\beta\left(f^{-1}(\lambda), r\right)\right](x)$ and $s \leq\left[f^{-1}(\delta(\mu, r))\right](y) \leq\left[\beta\left(f^{-1}(\mu), r\right)\right](y)$.

Hence, there exist $\nu=f^{-1}(\lambda), \rho=f^{-1}(\mu) \in I^{X}$ with $t \leq \beta(\nu, r)(x), s \leq \beta(\rho, r)(y) ; s, t \in$ $I_{0}$ so that $(t \wedge s)>\sup (\nu \wedge \rho)$, and thus $X$ is an $r$-fuzzy $\beta-T_{2}$. 
Proposition 2.4 Let $f: X \rightarrow Y$ be a surjective mapping. Assume that $\delta$ is a fuzzy operator on $Y$ such that

$$
f(\beta(\lambda, r)) \leq \delta(f(\lambda), r) \quad \forall \lambda \in I^{X}, \forall r \in I_{0} .
$$

Then, $X$ is an $r$-fuzzy $\beta-T_{i}$ implies that $Y$ is an $r$-fuzzy $\delta-T_{i}, \quad i=0,1,2$.

\section{Proof.}

Since $p \neq q$ in $Y$ implies that $x \neq y$ where $x=f^{-1}(p), y=f^{-1}(q)$ in $X$, and $X$ is an $r$-fuzzy $\beta$ - $T_{1}$, then there exists $\lambda \in I^{X}$ with $t \leq \beta(\lambda, r)\left(f^{-1}(p)\right) ; t \in I_{0}$ so that $t>\lambda\left(f^{-1}(q)\right)$, that is,

$$
t \leq[f(\beta(\lambda, r))](p) \leq[\delta(f(\lambda), r)](p) \text { and } t>(f(\lambda))(q),
$$

which means that there exists $\mu=f(\lambda) \in I^{Y}$ with $t \leq \delta(\mu, r)(p) ; t \in I_{0}$ so that $t>\mu(q)$. Hence, $Y$ is an $r$-fuzzy $\delta$-T, and consequently $Y$ is an $r$-fuzzy $\delta$ - $T_{0}$.

Now, for $p \neq q$ in $Y$ implies that $f^{-1}(p) \neq f^{-1}(q)$ in $X$ and $X$ is an $r$-fuzzy $\beta-T_{2}$, then there exist $\lambda, \mu \in I^{X}$ with $t \leq \beta(\lambda, r)\left(f^{-1}(p)\right), s \leq \beta(\mu, r)\left(f^{-1}(q)\right) ; s, t \in I_{0}$ so that $(t \wedge s)>\sup (\lambda \wedge \mu)$.

Since $\sup (\lambda \wedge \mu) \geq \sup (f(\lambda) \wedge f(\mu))$, then $(t \wedge s)>\sup (f(\lambda) \wedge f(\mu))$. Also,

$$
t \leq[f(\beta(\lambda, r))](p) \leq[\delta(f(\lambda), r)](p) \text { and } s \leq[f(\beta(\mu, r))](q) \leq[\delta(f(\mu), r)](q) .
$$

Hence, there exist $\nu=f(\lambda), \rho=f(\mu) \in I^{Y}$ with $t \leq \delta(\nu, r)(p), s \leq \delta(\rho, r)(q) ; s, t \in I_{0}$ so that $(t \wedge s)>\sup (\nu \wedge \rho)$, and thus $Y$ is an $r$-fuzzy $\delta-T_{2}$.

\section{Remark 2.1}

(1) For a fuzzy topological space $(X, \tau)$, by choosing $\beta=$ fuzzy interior operator, you can deduce the equivalence between the graded fuzzy separation axioms $(t, s)-T_{i}$, $i=0,1,2 ; t, s \in I_{0}$ introduced in $[3,4]$ and the axioms $r$-fuzzy $\beta$ - $T_{i}, i=0,1,2$.

(2) For two fuzzy topological spaces $(X, \tau),(Y, \sigma)$, and $f: X \rightarrow Y$ a mapping, by choosing $\beta=$ fuzzy interior operator, we get that $(X, \tau)$ is $(t, s)-T_{i}, i=$ $0,1,2 ; t, s \in I_{0}$ whenever $(Y, \sigma)$ is $(t, s)-T_{i}, i=0,1,2 ; t, s \in I_{0}$ and $f$ is injective fuzzy continuous (when $\delta=$ fuzzy interior operator in Proposition 2.3) as shown in [3]. This is equivalent to $f$ is injective and $\alpha=$ identity operator, $\beta=$ interior operator, $\delta=$ interior operator, $\theta=$ identity operator and $\mathcal{I}=\mathcal{I}^{\circ}$ in Definition $1.1(1)$.

(3) For two fuzzy topological spaces $(X, \tau),(Y, \sigma)$, and $f: X \rightarrow Y$ a mapping, by choosing $\delta=$ fuzzy interior operator, we get that $(Y, \sigma)$ is $(t, s)-T_{i}, i=$ $0,1,2 ; t, s \in I_{0}$ whenever $(X, \tau)$ is $(t, s)-T_{i}, i=0,1,2 ; t, s \in I_{0}$ and $f$ is surjective fuzzy open (when $\beta=$ fuzzy interior operator in Proposition 2.4) as shown in [3]. This is equivalent to $f$ is surjective and $\alpha=$ identity operator, $\beta=$ interior operator, $\delta=$ interior operator, $\theta=$ identity operator and $\mathcal{I}=\mathcal{I}^{\circ}$ in Definition $1.1(2)$. 


\section{3. $r$-fuzzy $\alpha$-connected spaces}

Here, we introduce the $r$-fuzzy connectedness of a space $X$ relative to a fuzzy operator $\alpha$. Assume (with respect to any fuzzy topology $\tau$ defined on $X$ ) that:

$$
\lambda \leq \alpha(\lambda, r) \leq \operatorname{cl}_{\tau}(\lambda, r) \quad \forall \lambda \in I^{X} ; r \in I_{0} .
$$

Also, assume that $\alpha$ is a monotone operator, that is,

$$
\mu \leq \nu \text { implies } \alpha(\mu, r) \leq \alpha(\nu, r) \forall \mu, \nu \in I^{X} ; r \in I_{0} .
$$

Definition 3.1 Let $X$ be a non-empty set. Then,

(1) the fuzzy sets $\lambda, \mu \in I^{X}$ are called $r$-fuzzy $\alpha$-separated sets if

$$
\alpha(\lambda, r) \wedge \mu=\lambda \wedge \alpha(\mu, r)=\overline{0} ; r \in I_{0}
$$

(2) $X$ is called $r$-fuzzy $\alpha$-connected space if it could not be found $\lambda, \mu \in I^{X}, \lambda \neq$ $\overline{0}, \mu \neq \overline{0}$ such that $\lambda, \mu$ are $r$-fuzzy $\alpha$-separated and $\lambda \vee \mu=\overline{1}$. That is, there are no $r$-fuzzy $\alpha$-separated sets $\lambda, \mu \in I^{X}$ except $\lambda=\overline{0}$ or $\mu=\overline{0}$.

Definition 3.2 Let $\lambda, \mu \in I^{X}, \lambda \neq \overline{0}, \mu \neq \overline{0}$ such that:

(1) $\lambda, \mu$ are $r$-fuzzy $\alpha$-separated and $\lambda \vee \mu=\overline{1}$. Then $X$ is called $r$-fuzzy $\alpha$ disconnected space.

(2) $\lambda, \mu$ are $r$-fuzzy $\alpha$-separated and $\lambda \vee \mu=\nu$. Then $\nu$ is called $r$-fuzzy $\alpha$ disconnected fuzzy set in $I^{X}$.

(3) $\lambda, \mu$ are $r$-fuzzy $\alpha$-separated and $\lambda \vee \mu=\chi_{A}, A \subseteq X$. Then $A$ is called $r$-fuzzy $\alpha$-disconnected crisp set in $I^{X}$.

Remark 3.1 For a fuzzy topological space $(X, \tau)$

(1) Taking $\alpha=$ fuzzy closure operator on $(X, \tau)$, then we have the $r$-fuzzy connectedness as given in [5].

(2) Taking $\alpha=$ fuzzy preclosure operator on $(X, \tau)$, then we have the $r$-fuzzy preconnectedness as given in [7].

(3) Taking $\alpha=$ fuzzy strongly semi-closure operator on $(X, \tau)$, then we have the $r$-fuzzy strongly connectedness as given in [6].

(4) Taking $\alpha=$ fuzzy semi-closure operator on $(X, \tau)$, then we have the 1-type of $r$-fuzzy strongly connectedness as given in [6]. 
(5) Taking $\alpha=$ fuzzy semi-preclosure operator on $(X, \tau)$, then we have the $r$-fuzzy semi-preconnectedness as given in [7].

(6) Taking $\alpha=$ fuzzy strongly preclosure operator on $(X, \tau)$, then we have the $r$-fuzzy strongly preconnectedness as given in [7].

Example 3.1 Let $X=\{x, y\}, r \in I_{0}$,

$$
\alpha(\nu, r)= \begin{cases}\nu & \text { at } \nu=\overline{0}, \overline{1} \\ x_{1} & \text { at } \overline{0}<\nu \leq x_{1} \\ y_{1} & \text { at } \overline{0}<\nu \leq y_{1} \\ \overline{1} & \text { otherwise }\end{cases}
$$

Now, at $\lambda \neq \overline{0}, \lambda \leq x_{1}, \mu \neq \overline{0}, \mu \leq y_{1}, r \leq \frac{1}{4}$, then we have $\alpha(\lambda, r) \wedge \mu=x_{1} \wedge \mu=\overline{0}$ and $\alpha(\mu, r) \wedge \lambda=y_{1} \wedge \lambda=\overline{0}$, and thus $\lambda, \mu$ are $r$-fuzzy $\alpha$-separated sets for $\lambda \neq \overline{0}, \lambda \leq$ $x_{1}, \mu \neq \overline{0}, \mu \leq y_{1}$.

At $\lambda=x_{1}$ and $\mu=y_{1}$, we get $r$-fuzzy $\alpha$-separated sets with $\overline{1}=\lambda \vee \mu$. Hence, $X$ is an $r$-fuzzy $\alpha$-disconnected space.

Proposition 3.1 Let $(X, \tau)$ be a fuzzy topological space. Then the following are equivalent.

(1) $(X, \tau)$ is r-fuzzy $\alpha$-connected.

(2) $\lambda \wedge \mu=\overline{0}, \tau(\lambda) \geq r, \tau(\mu) \geq r ; r \in I_{0}$, and $\overline{1}=\lambda \vee \mu \quad$ imply $\lambda=\overline{0}$ or $\mu=\overline{0}$.

(3) $\lambda \wedge \mu=\overline{0}, \tau_{c}(\lambda) \geq r, \tau_{c}(\mu) \geq r ; r \in I_{0}$, and $\overline{1}=\lambda \vee \mu \quad$ imply $\lambda=\overline{0}$ or $\mu=\overline{0}$.

Proof. $(1) \Rightarrow(2)$ : Let $\lambda, \mu \in I^{X}$ with $\tau(\lambda) \geq r, \tau(\mu) \geq r ; r \in I_{0}$ such that $\lambda \wedge \mu=\overline{0}$ and $\overline{1}=\lambda \vee \mu$. Then, $\lambda=\mu^{c}$ and $\mu=\lambda^{c}$, and then

$$
\begin{gathered}
\overline{0}=\lambda \wedge \mu=\mu^{c} \wedge \lambda^{c}=\operatorname{cl}_{\tau}\left(\mu^{c}, r\right) \wedge \lambda^{c} \geq \alpha\left(\mu^{c}, r\right) \wedge \lambda^{c} \text { and } \\
\overline{0}=\lambda \wedge \mu=\mu^{c} \wedge \lambda^{c}=\mu^{c} \wedge \operatorname{cl}_{\tau}\left(\lambda^{c}, r\right) \geq \mu^{c} \wedge \alpha\left(\lambda^{c}, r\right) ; r \in I_{0},
\end{gathered}
$$

which means that $\lambda^{c}, \mu^{c}$ are fuzzy $\alpha$-separated so that $\lambda^{c} \vee \mu^{c}=\mu \vee \lambda=\overline{1}$. But $(X, \tau)$ is $r$-fuzzy $\alpha$-connected implies that $\lambda^{c}=\overline{0}$ or $\mu^{c}=\overline{0}$, and thus $\lambda=\overline{0}$ or $\mu=\overline{0}$.

$(2) \Rightarrow(3)$ : Clear.

$(3) \Rightarrow(1):$ Let $\lambda, \mu \in I^{X}, \lambda \neq \overline{0}, \mu \neq \overline{0}$ such that $\lambda \vee \mu=\overline{1}$. Taking $\nu=\operatorname{cl}_{\tau}(\lambda, r)$ and $\rho=\operatorname{cl}_{\tau}(\mu, r) ; r \in I_{0}$, then $\nu \vee \rho=\overline{1}$ and $\tau_{c}(\nu) \geq r, \tau_{c}(\rho) \geq r ; r \in I_{0}$.

Now, suppose that (3) is not satisfied. That is, $\nu \neq \overline{0}, \rho \neq \overline{0}$ and $\nu \wedge \rho=\overline{0}$. Then,

$$
\begin{gathered}
\alpha(\lambda, r) \wedge \mu \leq \operatorname{cl}_{\tau}(\lambda, r) \wedge \operatorname{cl}_{\tau}(\mu, r)=\nu \wedge \rho=\overline{0} \text { and } \\
\alpha(\mu, r) \wedge \lambda \leq \operatorname{cl}_{\tau}(\lambda, r) \wedge \operatorname{cl}_{\tau}(\mu, r)=\nu \wedge \rho=\overline{0},
\end{gathered}
$$

which means that $\lambda, \mu$ are $r$-fuzzy $\alpha$-separated sets, $\lambda \neq \overline{0}, \mu \neq \overline{0}$ with $\lambda \vee \mu=\overline{1}$. Hence, $(X, \tau)$ is not $r$-fuzzy $\alpha$-connected space. 
Proposition 3.2 Let $X$ be a non-empty set and $\lambda \in I^{X}$. Then the following are equivalent.

(1) $\lambda$ is r-fuzzy $\alpha$-connected.

(2) If $\mu, \rho$ are $r$-fuzzy $\alpha$-separated sets with $\lambda \leq \mu \vee \rho$, then $\lambda \wedge \mu=\overline{0}$ or $\lambda \wedge \rho=\overline{0}$.

(3) If $\mu, \rho$ are $r$-fuzzy $\alpha$-separated sets with $\lambda \leq \mu \vee \rho$, then $\lambda \leq \mu$ or $\lambda \leq \rho$.

Proof. $(1) \Rightarrow(2)$ : Let $\mu, \rho$ be $r$-fuzzy $\alpha$-separated with $\lambda \leq \mu \vee \rho$, that is, $\alpha(\mu, r) \wedge \rho=\alpha(\rho, r) \wedge \mu=\overline{0} ; r \in I_{0}$ so that $\lambda \leq \mu \vee \rho$. Then, from that $\alpha$ is a monotone fuzzy operator, we get that

$\alpha((\lambda \wedge \mu), r) \wedge(\lambda \wedge \rho) \leq \alpha(\lambda, r) \wedge \alpha((\mu, r) \wedge(\lambda \wedge \rho)=(\alpha(\lambda, r) \wedge \lambda) \wedge(\alpha((\mu, r) \wedge \rho)=\lambda \wedge \overline{0}=\overline{0}$

and

$$
\alpha((\lambda \wedge \rho), r) \wedge(\lambda \wedge \mu) \leq(\alpha(\lambda, r) \wedge \lambda) \wedge(\alpha(\rho, r) \wedge \mu)=\lambda \wedge \overline{0}=\overline{0} ; r \in I_{0} .
$$

That is, $\lambda \wedge \mu$ and $\lambda \wedge \rho$ are $r$-fuzzy $\alpha$-separated sets so that $\lambda=(\lambda \wedge \mu) \vee(\lambda \wedge \rho)$. But $\lambda$ is $r$-fuzzy $\alpha$-connected implies that $(\lambda \wedge \mu)=\overline{0}$ or $(\lambda \wedge \rho)=\overline{0}$.

(2) $\Rightarrow$ (3): If $\lambda \wedge \mu=\overline{0}$, then $\lambda=\lambda \wedge(\mu \vee \rho)=\lambda \wedge \rho$, and thus $\lambda \leq \rho$. Also, if $\lambda \wedge \rho=\overline{0}$, then $\lambda=\lambda \wedge \mu$, and then $\lambda \leq \mu$.

$(3) \Rightarrow(1)$ : Let $\mu, \rho$ be $r$-fuzzy $\alpha$-separated sets such that $\lambda=\mu \vee \rho$. Then, from (3), $\lambda \leq \mu$ or $\lambda \leq \rho$. If $\lambda \leq \mu$, then $\rho=\lambda \wedge \rho \leq \mu \wedge \rho \leq \alpha(\mu, r) \wedge \rho=\overline{0}$. Also, if $\lambda \leq \rho$, then $\mu=\lambda \wedge \mu \leq \rho \wedge \mu \leq \alpha(\rho, r) \wedge \mu=\overline{0}$. Hence, $\lambda$ is $r$-fuzzy $\alpha$-connected.

Theorem 3.1 Let $f: X \rightarrow Y$ be a mapping such that

$$
\alpha\left(f^{-1}(\nu), r\right) \leq f^{-1}(\theta(\nu, r)) \quad \forall \nu \in I^{Y}, r \in I_{0},
$$

where $\alpha$ is a fuzzy operator on $X$ and $\theta$ is a fuzzy operator on $Y$. Then, $f(\lambda) \in I^{Y}$ is $r$-fuzzy $\theta$-connected if $\lambda \in I^{X}$ is $r$-fuzzy $\alpha$-connected.

Proof. Let $\mu, \rho \in I^{Y}, \mu \neq \overline{0}, \rho \neq \overline{0}$ be $r$-fuzzy $\theta$-separated sets in $I^{Y}$ with $f(\lambda)=\mu \vee \rho$. That is, $\theta(\mu, r) \wedge \rho=\theta(\rho, r) \wedge \mu=\overline{0} ; r \in I_{0}$. Then, $\lambda \leq f^{-1}(\mu) \vee f^{-1}(\rho)$, and

$$
\begin{aligned}
\alpha\left(f^{-1}(\mu), r\right) \wedge f^{-1}(\rho) & \leq f^{-1}(\theta(\mu, r)) \wedge f^{-1}(\rho) \\
& =f^{-1}(\theta(\mu, r) \wedge \rho) \\
& =f^{-1}(\overline{0})=\overline{0}, \\
& \\
\alpha\left(f^{-1}(\rho), r\right) \wedge f^{-1}(\mu) & \leq f^{-1}(\theta(\rho, r)) \wedge f^{-1}(\mu) \\
& =f^{-1}(\theta(\rho, r) \wedge \mu) \\
& =f^{-1}(\overline{0})=\overline{0} .
\end{aligned}
$$


Hence, $f^{-1}(\mu)$ and $f^{-1}(\rho)$ are $r$-fuzzy $\alpha$-separated sets in $X$ so that $\lambda \leq f^{-1}(\mu) \vee f^{-1}(\rho)$. But $\lambda$ is $r$-fuzzy $\alpha$-connected means, from (3) in Proposition 3.2, that $\lambda \leq f^{-1}(\mu)$ or $\lambda \leq f^{-1}(\rho)$, which means that $f(\lambda) \leq \mu$ or $f(\lambda) \leq \rho$. Thus, again from (3) in Proposition 3.2, we get that $f(\lambda)$ is $r$-fuzzy $\theta$-connected.

Corollary 3.1 (Theorem 2.12 in [5]) Let $\left(X, \tau_{1}\right),\left(Y, \tau_{2}\right)$ be two fuzzy topological spaces. If $f: X \rightarrow Y$ is a fuzzy continuous mapping and $\lambda \in I^{X}$ is $r$-fuzzy connected in $X$, then $f(\lambda)$ is an $r$-fuzzy connected in $Y$.

Proof. Let $\alpha=$ fuzzy closure operator and $\theta=$ fuzzy closure operator. Then, the result follows from Theorem 3.1.

Corollary 3.2 (Theorems $2.12,3.11$ in $[6])$ Let $\left(X, \tau_{1}\right),\left(Y, \tau_{2}\right)$ be two fuzzy topological spaces. Let $f:\left(X, \tau_{1}\right) \rightarrow\left(Y, \tau_{2}\right)$ be $S$-irresolute (resp. irresolute). If $\lambda \in I^{X}$ is $r$-fuzzy strongly connected (resp. 1-type of $r$-fuzzy strongly connected) in $X$, then $f(\lambda)$ is $r$-fuzzy strongly connected (resp. 1-type of $r$-fuzzy strongly connected) in $Y$.

Proof. Let $\alpha=$ fuzzy strongly semi-closure (resp. semi-closure) operator and $\theta=$ fuzzy strongly semi-closure (resp. semi-closure) operator. Then, the result follows from Theorem 3.1.

Corollary 3.3 Let $\left(X, \tau_{1}\right),\left(Y, \tau_{2}\right)$ be two fuzzy topological spaces. Let $f:\left(X, \tau_{1}\right) \rightarrow$ $\left(Y, \tau_{2}\right)$ be fuzzy semi-pre-irresolute. If $\lambda \in I^{X}$ is $r$-fuzzy semi-preconnected in $X$, then $f(\lambda)$ is $r$-fuzzy semi-preconnected in $Y$.

Proof. Let $\alpha=$ fuzzy semi-preclosure operator and $\theta=$ fuzzy semi-preclosure operator. Then, the result follows from Theorem 3.1.

Corollary 3.4 (Theorem 5.10 in [7]) Let $\left(X, \tau_{1}\right),\left(Y, \tau_{2}\right)$ be two fuzzy topological spaces. Let $f:\left(X, \tau_{1}\right) \rightarrow\left(Y, \tau_{2}\right)$ be fuzzy strongly pre-irresolute (resp. pre-irresolute). If $\lambda \in I^{X}$ is $r$-fuzzy s preconnected (resp. preconnected) in $X$, then $f(\lambda)$ is $r$-fuzzy s preconnected (preconnected) in $Y$.

Proof. Let $\alpha=$ fuzzy strongly preclosure (resp. preclosure) operator and $\theta=$ fuzzy strongly preclosure (resp. preclosure) operator. Then, the result follows from Theorem 3.1.

Corollary 3.5 Let $\left(X, \tau_{1}\right),\left(Y, \tau_{2}\right)$ be two fuzzy topological spaces. Let $f:\left(X, \tau_{1}\right) \rightarrow$ $\left(Y, \tau_{2}\right)$ be fuzzy semi-continuous (resp. precontinuous, strongly semi-continuous, strongly precontinuous and semi-precontinuous) mapping. If $\lambda \in I^{X}$ is 1-type of $r$-fuzzy strongly connected (resp. $r$-fuzzy preconnected, $r$-fuzzy strongly connected, $r$-fuzzy strongly preconnected and $r$-fuzzy semi-preconnected) in $X$, then $f(\lambda)$ is $r$-fuzzy connected in $Y$. 
Proof. Let $\alpha=$ fuzzy semi-closure (resp. preclosure, strongly semi-closure, strongly preclosure and semi-preclosure) operator and $\theta=$ fuzzy closure operator. Then, the result follows from Theorem 3.1 .

Proposition 3.3 Any fuzzy point $x_{t}, t \in I_{0}$ is $r$-fuzzy $\alpha$-connected, and consequently $x_{1} \forall x \in X$ is $r$-fuzzy $\alpha$-connected.

Proof. Clear.

Definition 3.3 Let $X$ be a non-empty set and $\lambda \in I^{X}$. Then, $\lambda$ is $r$-fuzzy $\alpha$ component if $\lambda$ is maximal $r$-fuzzy $\alpha$-connected set in $X$, that is, if $\mu \geq \lambda$ and $\mu$ is $r$-fuzzy $\alpha$-connected set, then $\lambda=\mu$.

Proposition 3.4 Let $\lambda \neq \overline{0}$ be $r$-fuzzy $\alpha$-connected in $X$ and $\lambda \leq \mu \leq \alpha(\lambda, r) ; r \in I_{0}$. Then, $\mu$ is $r$-fuzzy $\alpha$-connected.

Proof. Let $\nu, \rho$ be $r$-fuzzy $\alpha$-separated sets such that $\mu=\nu \vee \rho$. That is, $\alpha(\nu, r) \wedge \rho=$ $\alpha(\rho, r) \wedge \nu=\overline{0} ; r \in I_{0}$. Since $\lambda \leq \mu$, then $\lambda \leq(\nu \vee \rho)$. From $\lambda$ is $r$-fuzzy $\alpha$-connected, and from (3) in Proposition 3.2, we have $\lambda \leq \nu$ or $\lambda \leq \rho$. If $\lambda \leq \nu$, then

$$
\rho=\mu \wedge \rho \leq \alpha(\lambda, r) \wedge \rho \leq \alpha(\nu, r) \wedge \rho=\overline{0}
$$

If $\lambda \leq \rho$, then

$$
\nu=\mu \wedge \nu \leq \alpha(\lambda, r) \wedge \nu \leq \alpha(\rho, r) \wedge \nu=\overline{0} .
$$

Hence, $\mu$ is $r$-fuzzy $\alpha$-connected.

\section{Fuzzy $\alpha$-compact spaces}

This section is devoted to introduce the notion of $r$-fuzzy $\alpha$-compact spaces.

Definition 4.1 Let $(X, \tau)$ be a fuzzy topological space, $\alpha$ a fuzzy operator on $X$, and $\mu \in I^{X}, r \in I_{0}$. Then, $\mu$ is called $r$-fuzzy $\alpha$-compact if for each family $\left\{\lambda_{j} \in I^{X}\right.$ : $\left.\tau\left(\lambda_{j}\right) \geq r, j \in J\right\}$ with $\mu \leq \bigvee_{j \in J} \lambda_{j}$, there exists a finite subset $J_{0} \subseteq J$ such that $\mu \leq \bigvee_{j \in J_{0}} \alpha\left(\lambda_{j}, r\right)$

Remark 4.1 For a fuzzy topological space $(X, \tau)$ :

(1) if $\alpha$ = fuzzy identity operator, we get the $r$-fuzzy compactness as given in [9].

(2) if $\alpha=$ fuzzy closure operator, we get the $r$-fuzzy almost compactness as given in $[9]$. 
(3) if $\alpha=$ fuzzy interior closure operator, we get the $r$-fuzzy near compactness as given in [9].

(4) if $\alpha=$ fuzzy semi-closure (resp. preclosure, strongly semi-closure, strongly preclosure and semi-preclosure) operator, we get the $r$-fuzzy semi-compactness (resp. precompactness, strongly semi-compactness, strongly precompactness and semiprecompactness [8]).

Theorem 4.1 Let $(X, \tau)$ and $(Y, \sigma)$ be two fuzzy topological spaces, $\alpha$ a fuzzy operator on $X, \theta$ is a fuzzy operators on $Y$. If $f: X \rightarrow Y$ is fuzzy $\left(\alpha\right.$, int $\left._{\tau}, \theta, i d_{Y}, \mathcal{I}^{\circ}\right)$-continuous and $\mu \in I^{X}$ is $r$-fuzzy compact in $X$, then $f(\mu)$ is $r$-fuzzy $\theta$-compact in $Y$.

Proof. Let $\left\{\lambda_{j} \in I^{Y}: \sigma\left(\lambda_{j}\right) \geq r, j \in J\right\}$ be a family with $f(\mu) \leq \bigvee_{j \in J} \lambda_{j}$. Since $f$ is fuzzy $\left(\alpha, \operatorname{int}_{\tau}, \theta, i d_{Y}, \mathcal{I}^{\circ}\right)$-continuous, we get that there exists $\mu_{j}=\operatorname{int}_{\tau}\left(f^{-1}\left(\theta\left(\lambda_{j}, r\right)\right), r\right) \in$ $I^{X}$ with $\tau\left(\mu_{j}\right) \geq r \forall j \in J$ such that

$$
\alpha\left(f^{-1}\left(\lambda_{j}\right), r\right) \leq \mu_{j} \leq f^{-1}\left(\theta\left(\lambda_{j}, r\right)\right) .
$$

Also, since $f^{-1}\left(\lambda_{j}\right) \leq \alpha\left(f^{-1}\left(\lambda_{j}\right), r\right)$, then

$$
f^{-1}\left(\lambda_{j}\right) \leq \mu_{j} \leq f^{-1}\left(\theta\left(\lambda_{j}, r\right)\right)
$$

which means that

$$
\mu \leq \bigvee_{j \in J} f^{-1}\left(\lambda_{j}\right) \leq \bigvee_{j \in J}\left(\mu_{j}\right) \leq f^{-1}\left(\bigvee_{j \in J} \theta\left(\lambda_{j}, r\right)\right)
$$

that is, $\mu \leq \bigvee_{j \in J}\left(\mu_{j}\right)$. By $r$-fuzzy compactness of $\mu$, there exists a finite set $J_{0} \subseteq J$ such that $\mu \leq \bigvee_{j \in J_{0}}\left(\mu_{j}\right)$, and thus

$$
f(\mu) \leq \bigvee_{j \in J_{0}} f\left(\mu_{j}\right) \leq \bigvee_{j \in J_{0}} \theta\left(\lambda_{j}, r\right)
$$

and therefore $f(\mu)$ is $r$-fuzzy $\theta$-compact.

Corollary 4.1 $([8])$ Let $(X, \tau)$ and $(Y, \sigma)$ be two fuzzy topological spaces. Let $f$ : $X \rightarrow Y$ be a fuzzy continuous mapping and $\mu \in I^{X}$ an $r$-fuzzy compact set in $X$, then $f(\mu)$ is $r$-fuzzy compact in $Y$.

Proof. Let $\alpha=$ fuzzy identity operator on $X, \theta=$ fuzzy identity operator and $\mathcal{I}=\mathcal{I}^{\circ}$, then the result follows from Theorem 4.1.

Corollary $4.2([8])$ Let $(X, \tau)$ and $(Y, \sigma)$ be two fuzzy topological spaces. Let $f$ : $X \rightarrow Y$ be a fuzzy weakly continuous mapping $([10])$ and $\mu \in I^{X}$ an $r$-fuzzy compact set in $X$, then $f(\mu)$ is $r$-fuzzy almost compact in $Y$. 
Proof. Let $\alpha=$ fuzzy identity operator on $X, \theta=$ fuzzy closure operator and $\mathcal{I}=\mathcal{I}^{\circ}$, then the result follows from Theorem 4.1.

Corollary $4.3([8])$ Let $(X, \tau)$ and $(Y, \sigma)$ be two fuzzy topological spaces. Let $f$ : $X \rightarrow Y$ be a fuzzy almost continuous mapping ([11]) and $\mu \in I^{X}$ an $r$-fuzzy compact set in $X$, then $f(\mu)$ is $r$-fuzzy nearly compact in $Y$.

Proof. Let $\alpha=$ fuzzy identity operator on $X, \theta=$ fuzzy interior closure operator and $\mathcal{I}=\mathcal{I}^{\circ}$, then the result follows from Theorem 4.1 .

Corollary 4.4 Let $(X, \tau)$ and $(Y, \sigma)$ be two fuzzy topological spaces. Let $f: X \rightarrow Y$ be a fuzzy semi-continuous [12] (resp. precontinuous [10], strongly semi-continuous [13], strongly precontinuous [7] and semi-precontinuous [10]) mapping, and $\mu \in I^{X}$ an $r$-fuzzy compact set in $X$, then $f(\mu)$ is $r$-fuzzy semi-compact (resp. precompact, strongly semi-compact, strongly precompact and semi-precompact) in $Y$.

Proof. Let $\alpha=$ fuzzy identity operator on $X, \theta=$ fuzzy semi-closure (resp. preclosure, strongly semi-closure, strongly preclosure and semi-preclosure) operator and $\mathcal{I}=\mathcal{I}^{\circ}$, then the result follows from Theorem 4.1.

\section{References}

[1] A. P. Šostak, On a fuzzy topological structure, Suppl Rend. Circ. Math. Palermo Ser. II, 11 (1985), 89-103.

[2] A. A. Ramadan, Y. C. Kim and M. K. El-Gayyar, Smooth ideals, J. of Korean Institute of intelligent systems, 12 (1) (2002), 90-95.

[3] F. Bayoumi and I. Ibedou, $T_{i^{-}}$spaces, I, The Journal of the Egyptian Mathematical Society, 10 (2) (2002), 179-199.

[4] Ismail Ibedou, Graded fuzzy topological spaces, Journal of Cogent Mathematics, 3 (2016), 1-13.

[5] Y. C. Kim and Y. S. Kim, Connectedness in smooth fuzzy topological spaces, Far East J. Math. Sci. 5 (3) (2002), 165-180.

[6] Y. C. Kim, A. A. Ramadan and S. E. Abbas, Strongly connectedness in L-fuzzy topological spaces, J. of Fuzzy Mathematics, 12 (2) (2004), 479-491.

[7] S. E. Abbas, Fuzzy SP-irresolute functions, Chaos, Solitons and Fractals, 21 (2004), $1075-1085$.

[8] Y. C. Kim and S. E. Abbas, On several types of r-fuzzy compactness, J. of Fuzzy Mathematics, 12 (4) (2004), 827-844. 
[9] S. E. Abbas, Fuzzy super irresolute functions, IJMMS, 42 (2003), 2689-2700.

[10] Y. C. Kim, A. A. Ramadan and S. E. Abbas, Weaker forms of continuity on Šstak's fuzzy topology, Indian J. of Pure and Applied Math., 34 (2) (2003), 311-333.

[11] Y. C. Kim, A. A. Ramadan and S. E. Abbas, Some good dilutions of compactness in Šstak's fuzzy topology, J. of Fuzzy Mathematics, 11 (4) (2003), 933-944.

[12] A. A. Ramadan, S. E. Abbas and Y. C. Kim, Fuzzy irresolute mappings in smooth fuzzy topological spaces, J. of Fuzzy Mathematics, 9 (4) (2001), 865-977.

[13] S. E. Abbas, Fuzzy S-irresolute mappings, Appl. Math. Comp., 155 (2) (2004), 329343. 